\title{
Dynamics of undeforming regions in the lead up to failure: jumping scales from lab to field
}

\author{
Antoinette Tordesillas ${ }^{1 *}$, Shuo Zhou ${ }^{1}$, Lachlan Campbell ${ }^{2}$ and Pat Bellett ${ }^{2}$ \\ ${ }^{1}$ School of Mathematics and Statistics, University of Melbourne, Australia \\ ${ }^{2}$ GroundProbe, Orica Monitor, Australia
}

\begin{abstract}
Knowledge transfer from micromechanics of granular media failure to geohazard forecasting and mitigation has been slow. But in the face of a rapidly expanding data infrastructure on the motion of individual grains for laboratory samples - and ground motion data at the field scale - opportunities to accelerate this knowledge transfer are emerging. In particular, such data assets coupled with data-driven approaches enable 'new eyes' to re-examine granular failure. To this end, effective strategies that can jump scales from bench to field are urgently needed. Here we demonstrate one strategy that focusses on the study of deformation patterns in the precursory failure regime using kinematic data. Unlike previous studies which focus on regions of high strains, here we probe the development and evolution of near-undeforming regions through the lens of explosive percolation. We find a common dynamical signature in which undeforming regions, which are initially transient in the precursory failure regime, become persistent from the time of imminent failure. We demonstrate the robustness of these findings for data on individual grain motions in a classical laboratory test and ground motion in two real landslides at vastly different scales.
\end{abstract}

\section{Introduction}

Marcel Proust once said "The real voyage of discovery consists not in seeking new landscapes, but in having new eyes" [1]. Past studies of the micromechanics of deformation, aimed toward improving fundamental understanding of the precursory failure regime, have largely focused on the evolution of regions of high strain [2-8]. In this study, we not only cast new eyes on deformation, but we also focus on the opposite extreme. That is, we examine the evolution of zones of vanishing deformation in the lead up to, at incipient and during failure - at both laboratory and field levels. Data on the kinematics of a well-studied discrete element simulation of a planar biaxial compression test [6-9] and two open pit mine slopes are examined in search of a common dynamical signature [9-11]: see Biax, M1 and M2 in Fig. 1. The aim is to improve fundamental knowledge of granular failure and help advance data-driven tools for geohazard forecasting and mitigation (e.g., [9-11]).

Studies of near undeforming regions in granular media have mainly focused on so-called "dead zones" which develop around solid bodies and structures [1214]. By far the most well-known are those that emerge in soil-solid interaction studies where the soil is in contact with various bodies: foundation, tillage implements and penetromers, off-road vehicles and biophysical intruders (e.g., [13-14] and references therein). The dead zones may be static or may move in near rigid-body motion, sometimes at the same velocity as the solid body. In the classical problem of indentation by a flat punch, Prandtl [15] hypothesized the presence of a dead zone beneath the punch and various experiments have confirmed this for different granular materials (e.g., Biarez's experiments in Fig. 1a) [13].

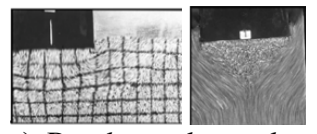

a) Dead zone beneath a punch from J. Biarez.

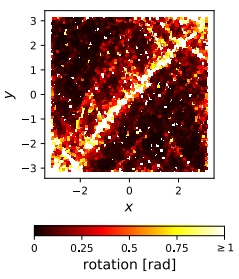

b) Shear band in Biax

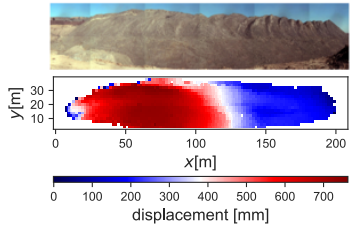

c) Slope M1 and landslide location

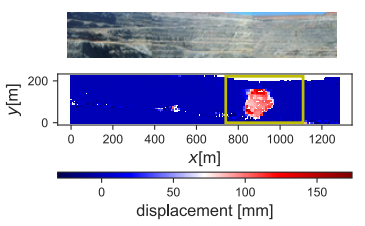

d) Slope $\mathbf{M 2}$ and landslide location
Fig. 1. Kinematics at failure across scales.

More generally, near rigid-body moving regions also emerge in geological hazards like landslides, such as when a rock mass begins to detach from the host rock and moves downslope under gravity [16], or near obstacles along the paths of earthflows and debris flows [17]. Yet, despite their prevalence in granular failure and flow, the dynamics of these endogenous structures, whether transient or persistent, remains largely unexplored. Here our objective is to identify and study the dynamics of undeforming regions throughout the

* Corresponding author: atordesi@unimelb.edu.au

A video is available at https://doi.org/10.48448/sgfr-dm36 
precursory failure regime from displacement data on systems with vastly different spatial scales: individual grain motion in a biaxial compression test and ground motion radar data on a landslide in two slopes, one spanning hundreds of meters while the other is over a kilometer wide. To the best of our knowledge, this is among the first efforts which directly relate granular micromechanics to the monitoring of a developing landslide using state-of-the-art remote sensing data.

\section{Data}

Three data sets, labeled Biax, M1 and M2, are examined. Biax has been characterized and reported in many papers (e.g., [6-10]), while M1 and M2 are described in [9-10]. Let $\boldsymbol{n}$ be the total number of observation points in the studied system: grains in Biax and pixel locations in $\mathbf{M i}(i=1,2)$. Biax is a discrete element simulation of an assembly of $\boldsymbol{n}=\mathbf{5 0 9 8}$ polydisperse spherical grains, submitted to planar biaxial compression test, under constant confining pressure [6]. A total of $\boldsymbol{N}=\mathbf{1 5 0}$ equilibrium strain states, labelled $\boldsymbol{t}=\mathbf{1}, 2, \ldots, \mathbf{1 5 0}$, are analyzed. Global dilatation initiates at $\boldsymbol{t}=\mathbf{5 0}$. Force chains collectively buckle from around $\boldsymbol{t}=\mathbf{9 8}$, in turn triggering a brief period of strain softening that culminates in a fully formed shear band at $\boldsymbol{t}_{\boldsymbol{F}}=\mathbf{1 0 4}$. The micromechanics of this initially homogeneous sample has been comprehensively studied against various experiments in 2D and 3D (e.g., photoelastic disks [8] and sand [7-8]).

M1 and M2 are time series data from two developing rockslides [9-10]. These landslides occurred in two separate operational open pit mines. The mine operation, location and year of the rockslides are confidential. Ground-based slope stability radar technology was used to deliver continuous 1D measurements of line-of-sight (LOS) surface movement of the rock slope at sub-millimeter precision; consecutive time measurements are 6 minutes apart. Slope M1 is an unconsolidated material which stretches to around $200 \mathrm{~m}$ in length and $40 \mathrm{~m}$ in height (Fig. 1). A total of $n=1803$ pixel locations, spread across the entire slope, were monitored for a period of $N=4000$ time states: 17 days, from 10:07 May 31 to 9:06 June 17. On June 15, a rockslide occurred on the western side of the slope with an arcuate back scar and a strike length of around $120 \mathrm{~m}$. The global peak velocity is $0.56 \mathrm{~mm} / \mathrm{min}$ which was recorded at $t_{F}=3568$ (13:10 June 15): we refer to $t_{F}$ as the time of failure. A "competing slide" emerged to the southeast region. This second region of instability intermittently developed large movements, but self-stabilized the day before the collapse of the west wall. The same phenomenon is observed in laboratory tests on sand samples where competing shear bands sporadically form just before failure but which later disappear to give way to the "winning" so-called persistent pattern of shear band(s) in the failure regime [2-4]. The studied area of M2, spanning $1.28 \mathrm{~km}$ wide and around $224 \mathrm{~m}$ high, was monitored for $N=1355$ time states: approximately 6 days from 15:39 August 19, until 07:05 August 25, across $n=5394$ pixel locations. A rockslide occurred on the southeast wall at $t_{F}=$
1315 (03:00 August 25). Here, without loss of generality, we present results for a smaller 2-part region $\boldsymbol{S}+\mathcal{F}$, where part $\mathcal{F}$ is the region of catastrophic failure (source area of 1 million tonnes of collapsed rock material) and part $\boldsymbol{S}$ is the immediate surrounds of $\mathcal{F}$. Note that a separate study of this data has shown that $\boldsymbol{S}$ sufficiently captures the dynamics of deformation of the rest of the monitored region which did not collapse [10].

\section{Method}

We adopt the explosive percolation analysis in [9], inspired by the work in [18]. Different from [9], however, here we focus our attention on the undeforming regions, namely groups of points which form connected clusters in both physical space and kinematic space. We call these the physical-kinematic clusters $C$. Identification and analysis of the dynamics of $C$ proceed in three steps.

In Step 1, we map the data to the kinematic state space $\Omega$ that summarizes the motion in the form of a point pattern $\wp$. Each point in $\wp$ is described by its displacement vector $\left(u_{x}, u_{y}\right)$ in Biax; or by the 1dimensional LOS displacement $(u)$ in the case of M1 and M2. Each strain state $t$ of the loading period in Biax has a corresponding $\wp(t), t \in[1,150]$; the same applies for every time state $t$ of the monitoring campaign $t \in$ $[1,3568]$ for $\mathbf{M 1}$ and $t \in[1,1355]$ for $\mathbf{M} 2$.

In Step 2, we generate the order parameter $p(r, t)$ vs. $r$ profiles for each $\wp(t)$. In essence, this profile identifies the dominant or largest components in $\wp(t)$, as well as summarizes their dynamics. We limit our attention to clusters that are of size greater than $10 \%$ of $n$. Clusters in $\wp(t)$ are groups of near-colocated points in $\Omega$, namely, pixels with very similar motion. To find these clusters, points within a kinematic-distance $r$ in $\Omega$ are classified in the same cluster. The growth in the size of the largest cluster, $p(r, t)=G(r, t) / n$, is tracked as $r$ is systematically increased, such that $G(r, t)$ is the number of points in the largest cluster. In the absence of a clustering pattern in $\Omega, p(r, t)$ should increase with $r$ continuously until the largest cluster contains all $n$ points. On the other hand, as time advances towards failure and as strain localization patterns begin to form, member points may form transient undeforming clusters: groups of grains/pixels which briefly move in near-rigid motion. Such partitioned motions result in multiple discontinuous jumps in $p(r, t)$ as $r$ increases. Each such jump or "explosive" growth in the size of the largest cluster results when a cluster is amalgamated into the largest cluster. These mergers lead to a distinctive stair-case pattern of consecutive "run-rise" cycles in the profile $p(r, t)$ vs. $r$. The height of each "rise" corresponds to the size of the newly merged cluster, while the width of each "run" preceding the rise corresponds to the kinematic separation, the difference between the mean displacements of points in the largest cluster and its newly subsumed members. The width of the last run or plateau that result in a system-spanning cluster increases as failure draws near. In M1 and M2, this corresponds to the active region beginning to detach 
from the host rock and accelerate downslope [9].

In Step 3, we find the set of physical-kinematic clusters $C(t)$ in $\wp(t)$. We map each dominant kinematic cluster from Step 2 into the physical network: contact network of Biax or the radar grid of $\mathbf{M i}$, to see which of these also form clusters in physical space. That is, we identify in the physical network of $\mathbf{M} \boldsymbol{i}$ those nodes from the dominant kinematic clusters and eliminate all other nodes in this physical network. In general, this results in a multi-component graph, out of which we select the largest components. For the cases studied here, two physical-kinematic clusters $C(t)$ emerge: these essentially share the same membership as the dominant kinematic clusters from Step 2. That is, kinematic clusters reveal collective motion in the physical body. To quantify their dynamics, the time evolution of the Jaccard index $J, 0 \leq J \leq 1$, a measure of the similarity of the clusters $C(t)$ across two consecutive time states, is examined to determine transience or persistence of clusters $C(t)$ in the lead up to and during failure. $J=$ $1(J=0)$ means the clusters at two consecutive time states have the same (no common) member points.

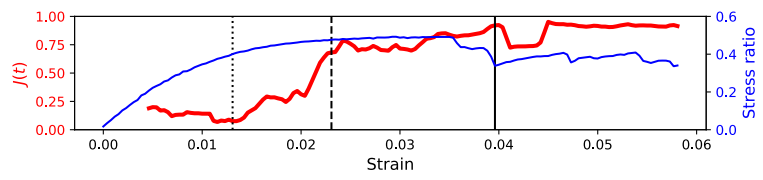

a) Biax

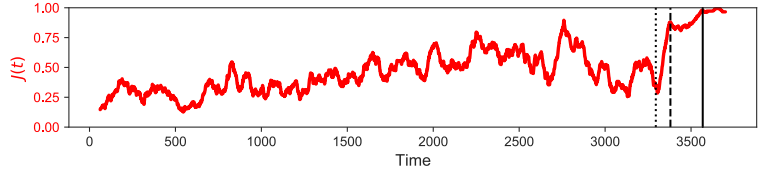

b) M1

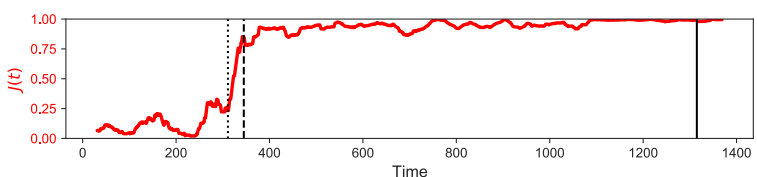

c) $\mathbf{M} 2$

Fig. 2. Time evolution of the Jaccard index $J$. For Biax, the stress ratio is included and the axial strain states correspond to the analyzed time states. The initial transition state, $t_{i}$ (dotted line), is the state from which the clustering persistence rapidly increases. The earliest state when the clustering pattern stabilizes, $t^{*}$ (dashed line), may be used as an indicator of imminent failure. Time of failure is $t_{F}$ (solid line).

\section{Results}

The evolution of undeforming regions in the pre-failure regime is shown in Figs. 2-5. In the early stages $t<t_{i}$, no persistent clustering manifests in the systems (Fig. 2). The interaction between the two competing slides in M1 leads to pronounced fluctuations in $t<t_{i}$ (Fig. 2b). That said, M1 shows relatively better clustering persistence across $t<t_{i}$ compared to Biax and M2 where $J$ is around 0.25 or less (Fig. 2a,c). The visual of $\wp(t)$ at $t_{i}$ in the kinematic space (Fig. 3a) shows that the kinematic clusters in the early time states are still either spread-out in $\Omega$ as is the case in Biax, or, are obscured by the relatively large number of noise points in $\mathbf{M 1}$ and M2. Note that noise from various perturbations like blasting, drilling, weather conditions and truck movements are present since these are operational mines. But since we are interested in methods that are robust to noise, we did not perform any filtering or preprocessing of the data for M1 and M2.
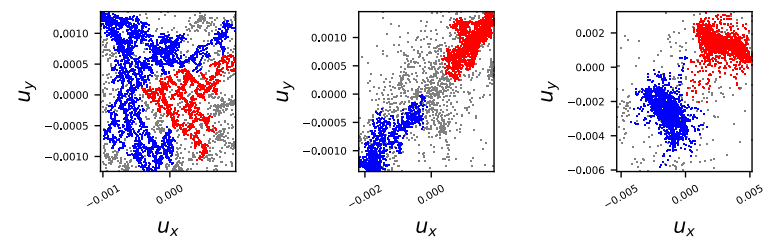

a) Biax at $t_{i}=35, t^{*}=61$, and $t_{F}=104$
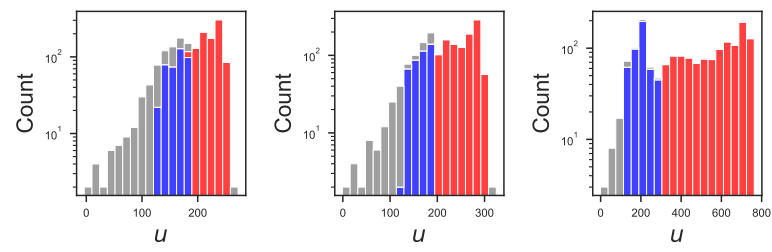

b) M1 at $t_{i}=3295, t^{*}=3380$, and $t_{F}=3568$
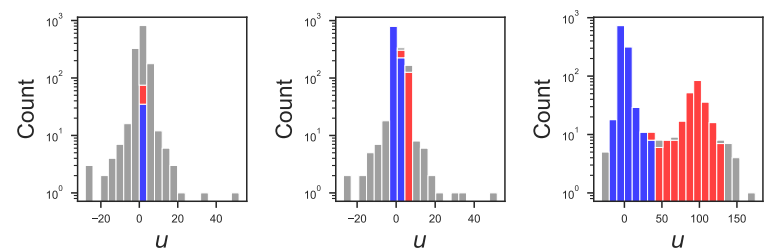

c) $\mathbf{M} 2$ at $t_{i}=311, t^{*}=345$, and $t_{F}=1315$

Fig. 3. Development of two clusters in kinematic state space $\Omega$. Kinematic separation and compactness of clusters increase in Biax as $t \rightarrow t_{F}$. Since $\Omega$ is 1D in M1 and M2, a frequency histogram of $u$ is more informative than a visual of $\wp(t)$. Red dots in (a) or bars in (b,c) correspond to points in the fastestmoving cluster; gray dots or bars are non-cluster points.

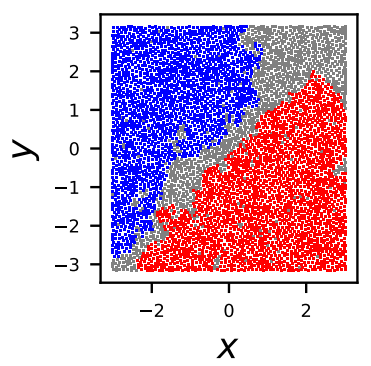

a) $\operatorname{Biax} a t t^{*}=61$

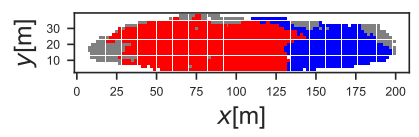

b) $\mathbf{M 1}$ at $t^{*}=3380$

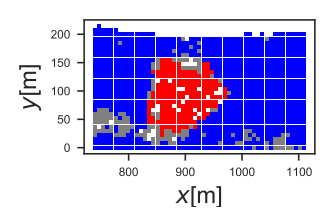

c) $\mathbf{M} 2$ at $t^{*}=345$
Fig. 4. The physical-kinematic clusters $C\left(t^{*}\right)$ in the physical space. The fastest-moving cluster (red) is the "active" or high landslide risk region in M1 and M2. Gray grains or pixels do not belong to the kinematic clusters.

As failure draws near, the clustering persistence starts to increase rapidly from $t_{i}$ until $t^{*}$, from which $J(t)$ remains high above 0.75 . This suggests that the clustering patterns in the ensuing states are highly similar to the pattern identified at $t^{*}$, such that an early prediction of the impending failure region is now possible (Figs. 2-4). In all systems, two dominant clusters emerged over $t \geq t^{*}$. In Biax, $t^{*}=61$ is well before peak stress $(t=98)$ and $t_{F}=104$. In M1, $t^{*}=$ $3380=18: 05$ June 14 is about 19 hours in advance of the time of collapse. In M2, the failure region can be 
predicted at $t^{*}=345=02: 03$ August 21, which is over 4 days prior to the collapse on 03:00 August 25. A further confirmation of the prediction at $t^{*}$ can be seen in the increasing kinematic separation of the clusters in $\wp$ (Fig. 3). Also, we can compare Fig. 4 to the actual failure location in Fig. 1. The adjacent boundaries of the kinematic clusters in the physical space in Biax matches well the shear band boundaries: compare Fig. 4a to Fig. 1b. The red active cluster for M1 and M2 in Fig. 4b,c accurately predicts the location and geometry of the landslides in Fig. 1c,d, respectively.

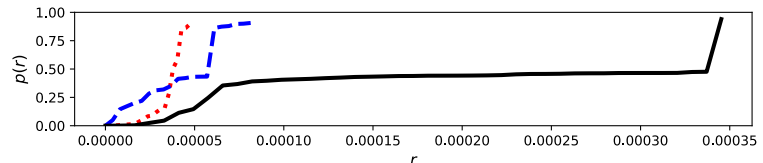

a) Biax

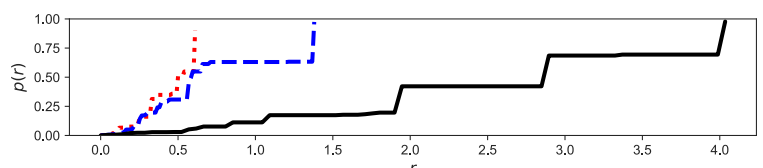

b) M1

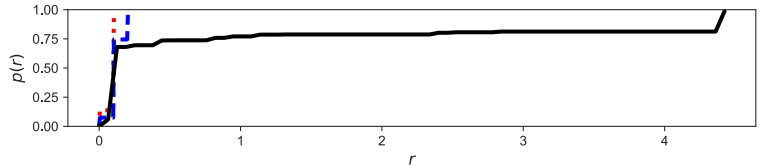

c) $\mathbf{M} 2$

Fig. 5. The order parameter $p(r) v s . r$ profiles at different strain or time states: $t_{i}$ (red dotted line), $t^{*}$ (blue dashed line), and $t_{F}$ (black solid line).

The kinematic separation of the clusters can also be seen in the order parameter $p(r)$ (Fig. 5). At $t_{i}$, when the impending failure location is first predicted, we observe a continuous transition to a system-spanning component at a small value of $r$. Closer to failure, a long plateau in $p(r)$ develops during which increases in $r$ merely lead to the addition of no links or intra-cluster links in $G(r, t)$. An inter-cluster link finally forms at a large value of $r=r_{\text {crit }}$, where the largest cluster merges with the next largest cluster to form a system-spanning component. In [9], it was shown that the time evolution of $r_{\text {crit }}$ correlates well with energy dissipation for Biax and manifests a sharp burst to a peak in the strain softening regime. Such an increasingly delayed transition is similar to what is observed in explosive transitions by D'Souza and Nagler [19].

\section{Conclusion}

The evolution of undeforming regions in a granular system is examined using a newly developed datadriven method based on the concept of explosive percolation. Kinematic data from three distinct systems are studied. One comprises individual grain motions from a simulation of a classical laboratory test in which the granular material is driven to failure, the other two are measurements of surface ground motion from ground-based radar monitoring of a developing landslide. Results demonstrate common aspects of dynamics in these vastly different systems. Ongoing work is now focused on more advanced methods that not only identify quasi-undeforming regions but quantify the dynamics of their interactions with different strain localization modes, such as microbands and shear bands, in confined as well as partially confined systems (e.g., retaining walls) across scales. We hope this work paves the way for studies that can jump scales and bridge the gap between fundamental micromechanics of granular failure and data-driven methods for forecasting and mitigation of geohazards like landslides.

\section{References}

[1] M. Proust, À la recherche du temps perdu (1923)

[2] K.A. Alshibli, S. Sture, J. Geotech. Geoenviron. Eng. 126, 495 (2000)

[3] J. Desrues, G. Viggiani, Int. J. Numer. Anal. Meth. Geomech. 28, 279 (2004)

[4] A.L. Rechenmacher, J. Mech. Phys. Solids 54, 22 (2006)

[5] A. Le Bouil, A. Amon, S. McNamara, J. Crassous, Phys. Rev. Lett. 112, 246001 (2014)

[6] A. Tordesillas, Phil. Mag. 87, 4987 (2007)

[7] A. Tordesillas, D.M. Walker, E. Andò, G. Viggiani, Proc. Roy. Soc. Lond. Ser. A 469, 20120606 (2013)

[8] D.M. Walker, A. Tordesillas, J. Zhang, R.P. Behringer, E. Andò, G. Viggiani, A. Druckrey, K. Alshibli, Int. J. Solids Struct. 54, 20 (2015)

[9] K. Singh, A. Tordesillas, Entropy 22, 67 (2020)

[10] A. Tordesillas, S. Kahagalage, L. Campbell, P. Bellett, E. Intrieri, R. Batterham, Sci. Rep. (in review)

[11] A. Tordesillas, S. Zhou, F. Di Traglia, E. Intrieri, New insights into the spatiotemporal precursory failure dynamics of the 2017 Xinmo landslide and its surrounds, in Understanding and Reducing Landslide Disaster Risk, edited by N. Casagli, V. Tofani, K. Sassa, P.T. Bobrowsky, K. Takara, WLF 2020, Springer, Cham (2020)

[12] G.B. Crosta, P.S. Powers, W.Z. Savage, A Study of Flow Development in Mass Movements of Granular Materials, in Open-File Report 91-383, Dept. of the Interior, US Geol. Survey (1991)

[13] J. Biarez, K. Wiendick, C. R. Acad. Sci. 256, 1217 (1963)

[14] A. Tordesillas, J. Shi, Proc. Roy. Soc. Lond. Ser. A 455, 261 (1999)

[15] L. Prandtl, Math. Phys. Kl., 1920, 74 (1920)

[16] A.M. Puzrin, A. Schmid, Géotechnique 62, 491 (2012)

[17] Y.J. Jiang, X.Y. Fan, L.J. Su, S.Y. Xiao, J. Sui, R.X. Zhang, Y. Song, Z.W. Shen, Landslides (2020)

[18] D. Achlioptas, R.M. D'Souza, J. Spencer, Science 323, 1453 (2009)

[19] R.M. D'Souza, J. Nagler, Nat. Phys. 11, 531 (2015). 